\title{
Aplicación de la metodología ApS en estudiantes de Finanzas. Valoración desde la perspectiva del estudiante y de la entidad receptora de la ApS
}

\author{
Begoña Cabanés-Cacho ${ }^{a}$, Nieves García-Casarejos ${ }^{b}$ e Israel Romera \\ ${ }^{a}$ Facultad de Economía y Empresa, Universidad de Zaragoza, bcabanes@unizar.es ${ }^{\text {b} F a c u l t a d ~ d e ~}$ \\ Economía y Empresa, Universidad de Zaragoza, ngarcia@unizar.es y ${ }^{\mathrm{C}}$ Facultad de Economía y \\ Empresa, Universidad de Zaragoza, iromera@unizar.es
}

\begin{abstract}
This project uses the Learning-service methodology, which combines the acquisition of skills with the service for social organizations, and applies it to the students of a subject in the Degree "Finance and Accounting". From its application is expected to achieve benefits for both parties, students and entities receiving the service.

Specifically, the aim of the Learning-service is to provide greater attention to the personal abilities and communication and professional skills that need to be achieved by the student of this Degree; and, on the other hand, to know the perception of the participating entities that receive the service in order to detect their satisfaction with the activity that is being carried out.

The spirit that guides the realization of this activity is no other than to train future professionals in the field of finance in values such as ethics, sustainability, transparency, equity and solidarity, that are such necessary nowadays.
\end{abstract}

Keywords: finance, values, Learning-service, social organizations, competencies, integral training, receiving entity, valuation.

\footnotetext{
Resumen

Este trabajo utiliza la metodología ApS, que combina la adquisición de competencias con el servicio a las organizaciones sociales, y lo aplica a los alumnos de una asignatura del Grado en Finanzas y Contabilidad. De su aplicación se espera conseguir beneficios para ambas partes, alumnos y entidades receptoras del servicio. En concreto, se trata de prestar mediante la metodología ApS una mayor atención a las competencias y habilidades personales, comunicativas y profesionales a alcanzar por parte del estudiante del Grado en Finanzas; y, por otro, conocer la percepción de las entidades participantes receptoras del servicio para detectar su satisfacción con la actividad realizada.

El espíritu que guía la realización de esta actividad no es otro que formar a los futuros profesionales del ámbito de las finanzas en valores como la ética, sostenibilidad, transparencia, equidad y solidaridad, tan necesarios en la actual sociedad.
} 
Aplicación de la metodología ApS en estudiantes de Finanzas. Valoración desde la perspectiva del estudiante y de la entidad receptora de la ApS

Palabras clave: finanzas, valores, ApS, organizaciones sociales, competencias, formación integral, entidad receptora, valoración.

\section{Introducción}

El proyecto nace con la finalidad de transferir a organizaciones y entidades, las competencias y habilidades alcanzadas por los estudiantes del grado en Finanzas y Contabilidad de la Universidad de Zaragoza, obligándoles a salir de las aulas, de su zona de confort y acercarse a colectivos con los que, habitualmente, no mantienen ningún tipo de relación, para poner en valor lo aprendido en el aula.

Para ello, además de centramos en conocer qué competencias alcanzan a través de la actividad de Aprendizaje-Servicio (en adelante ApS), así como la satisfacción del estudiante con la actividad, nos planteamos valorar cómo perciben este tipo de actuaciones las entidades receptoras de la ApS.

La actividad de ApS supone para los alumnos una experiencia transformadora, ya que parten de una situacion de conocimiento teorico pero de desconocimiento, en muchas ocasiones, de la realidad. Cuando realizan el servicio se producen en el estudiante nuevos pensamientos y sentimientos que le ayudan a reflexionar sobre su responsabilidad en el mundo, que sólo se podrá producir si el alumno tiene sensibilidad hacia las necesidades sociales que le rodean (Wong, 2007; Perez Gómez, 2010; Chiva-Bartoll, Pallarés-Piquer y Gil-Gómez, 2018).

En este contexto, la Universidad mediante estas ApS actúa de nexo de unión entre la enseñanza universitaria, que pretende una formación integral del alumno, y su entorno, la sociedad entendida en sentido amplio, que no está únicamente formada por entidades mercantiles sino también por entidades pertenecientes al tercer sector, ENL, ONGs, empresas de economía social, etc. Asimismo, esperamos que mediante la prestación de este servicio, las entidades, organizaciones o asociaciones potencien sus vínculos con la Universidad (Saz y Ramo, 2015).

El interés de este trabajo, aplicado a los estudiantes de la titulación de Finanzas y Contabilidad de la Facultad de Economia y Empresa, se justifica ya que: por un lado, permite incorporar prácticas que fomentan el desarrollo de valores sociales en los alumnos de una titulación con un alto contenido y visión economicista; por otro, consigue transmitir a la sociedad (a través de sus asociaciones, organizaciones no gubernamentales, instituciones educativas) parte de las competencias de responsabilidad social adquiridas en el aula, consiguiendo de este modo un efecto divulgativo, en consonancia con la Estrategia Europea 2020 que anima a difundir la RSE como herramienta para contribuir a la mejora de la competitividad de la economía de forma sostenible y con cohesión social.

Respecto al desarrollo de valores sociales, las ApS contribuyen a la formacion integral de los estudiantes, ya que el compromiso social que supone la actividad, genera un efecto sobre las personas hacia una actitud más social y responsable (Saz y Ramo, 2015).

La adquisición de competencias, es decir, las habilidades, actitudes, valores y conocimientos, tienen lugar como parte de un proceso de interconexióncon las formas de

(cc)) BY-NG-ND 2018, UniversitatPolitècnica de València

Congreso IN-RED (2018) 
ser, pensar, sentir y ver que caracterizan al grupo y al entorno en el que se desenvuelve nuestra vida (Lave y Wenger, 1991), y que se corresponde con los cuatro ejes del aprendizaje que recoge UNESCO: aprender a conocer, aprender a hacer, aprender a ser y aprender a convivir (Delors, 1996). Pero todo aprendizaje, en particular aquel que es relevante y duradero (Peréz Gómez, 2010), es fruto de la participación del individuo en prácticas sociales, por ser miembro de una comunidad social (Peréz Gómez y Soto, 2009) Este conocimiento lo construyen los estudiantes interpretando, analizando y evaluando, y no simplemente recitando información (Daniels y Bizar, 2005).

Además, para que el aprendizaje se produzca es necesario un alto grado de implicacion e interes por parte de los alumnos. Estos deben poder aplicar y consolidar las competencias adquiridas en el aula a una situación real (Gil-Gómez et al., 2016; Rodríguez, 2014).

Así, de los 39 matriculados en la asignatura optativa de "Gobierno corporativo y Responsabilidad Social”, 32 estudiantes aceptaron realizar esta actividad voluntaria, lo que supone un $82 \%$ del total.

\section{Objetivos}

Son dos los colectivos a los que se pretende impactar con este trabajo. Por un lado, a los estudiantes de la asignatura en la que se realiza la ApS y, por otro, a las entidades receptoras de la $\mathrm{ApS}$.

En el colectivo de estudiantes se pretende mejorar las siguientes competencias transversales:

1. Habilidades profesionales (resolución de problemas, organización, análisis y procesamiento de información, tecnológicas...).

2. Habilidades comunicativas (escucha activa, asertividad, comunicación verbal, comunicación no verbal, ...).

3. Habilidades personales (autoestima, seguridad, inteligencia emocional, proactividad, ...).

En el colectivo de las entidades receptoras de la ApS, se pretende:

1. Que conozcan mejor las competencias de los graduados en Finanzas de la Universidad de Zaragoza, y puedan valorar el impacto en la organización tras el servicio.

2. Conocer cual es su percepción del servicio, es decir, lo que les ha aportado participar en el proyecto.

3. Identificar su grado de satisfacción y necesidades futuras. 
Aplicación de la metodología ApS en estudiantes de Finanzas. Valoración desde la perspectiva del estudiante y de la entidad receptora de la ApS

\section{Desarrollo de la innovación}

El proyecto se lleva a cabo en la asignatura "Gobierno Corporativo y RSE” de cuarto del grado en Finanzas y Contabilidad en la Facultad Economía y Empresa durante el curso 2016-17, y el resumen de su ficha técnica se recoge en la Tabla 1. El proyecto tiene carácter voluntario para los estudiantes que optan por la modalidad de evaluación continua puesto que se recoge en la guía docente con un peso del 15\% de la nota final.

Tabla 1. Ficha técnica y contextualizacion de las Aps realizadas

\begin{tabular}{|c|c|}
\hline \multicolumn{2}{|c|}{$\begin{array}{c}\text { Contextualización de la ApS } \\
\text { Ficha Técnica }\end{array}$} \\
\hline Estudiantes matriculados en la asignatura & 39 \\
\hline Alumnos evaluación continua & 32 \\
\hline Alumnos que realizan ApS & 32 \\
\hline Número de ApS realizadas & 6 \\
\hline Media de estudiantes por grupo & 4 \\
\hline Método de selección de la ApS & Por el estudiante \\
\hline Grado de implicación del estudiante & Alto (en media) \\
\hline Edad de los estudiantes & $>20$ años \\
\hline Participación por género & $56 \%$ hombres; $44 \%$ mujeres \\
\hline
\end{tabular}

Fuente: Elaboración propia

La Metodología ApS permite al estudiante profundizar en su aprendizaje realizando un servicio a la comunidad. Además, desde un punto de vista docente permite llevar a cabo una práctica innovadora que los alumnos realizarán fuera del aula y pondrán en práctica las competencias adquiridas en la asignatura en una actividad de utilidad para una organización en particular, un colectivo o la sociedad en general, y que se espera que tenga un impacto formativo y transformador en los participantes, en la institución educativa y en la comunidad (Mayor, 2012)

Para ello, deberán planificar la actividad, desarrollarla fuera de las aulas y evaluar los resultados obtenidos de la misma, exponiéndolos ante del resto de compañeros. Previo a la planificación, se consensuará con el profesor la actividad a realizar. Los estudiantes organizan un calendario, del cual hará un seguimiento el profesor en base a tutorías grupales, así como un informe y exposición al final del semestre para transmitir el conocimiento y experiencia adquirida al resto de sus compañeros que servirá para poder evaluar el trabajo realizado.

A continuación, se describen los proyectos de aprendizaje-servicio realizados:

- Colaborar con la Oficina Verde la Universidad de Zaragoza. A petición de la Oficina Verde se realizaría una encuesta e informes para mejorar la prestación de servicios en el Campus Río Ebro en dos aspectos: temperatura de confort en los edificios y movilidad sostenible. Esta actividad se espera que tenga una repercusión positiva y mejore la conciencia medioambiental en el Campus Río Ebro.

(cc)) EY-Nc-ND 2018, UniversitatPolitècnica de València 
- Fundacion Federico Ozanam. . La actividad consistirá en que nuestros alumnos impartirán una charla sobre reciclado y uso responsable de los materiales para sensibilizar a a los alumnos de sus centros de formación ocupacional.

- Juego de rol, “Ethica”. Se trata de un juego diseñado para explorar el impacto social y ambiental de las actividades bancarias, las inversiones y los negocios, y aprender a tomar decisiones más responsables y éticas en cuanto a la gestión del dinero. Los alumnos explicarán el juego a alumnos de bachillerato del Instituto Grande Covián y llevaran a cabo alguna partida, ayudándoles a desarrollar un espíritu crítico cuando tomen decisiones de inversión.

- Videos explicativos sobre conceptos básicos de finanzas. Estos videos pretenden difundir al gran público cuestiones básicas a las que todo ciudadano se tiene que enfrentar en su relación con entidades financieras.

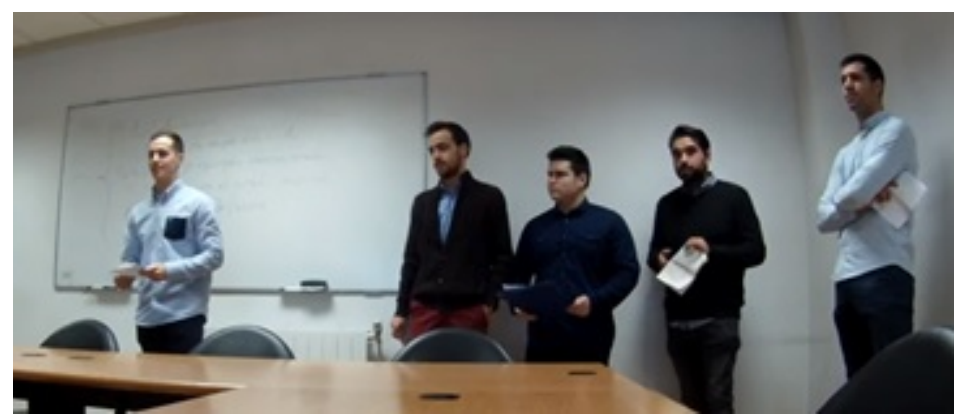

Fig. 1 Estudiantes de la asignatura realizando su ApS

Se supervisa y se lleva a cabo un seguimiento a través de tutorías, email, llamadas, etc. Al final del semestre cada grupo tendrá que explicar cuál ha sido su proyecto y que han aprendido al resto de sus compañeros. Para el seguimiento se utilizan, semanalmente, 10 minutos de clase, para hablar con los miembros de cada grupo y establecer las citas para tutorias, entrega de actividades parciales, etc. Cada grupo esta formado por, entre 3 y 6 personas.

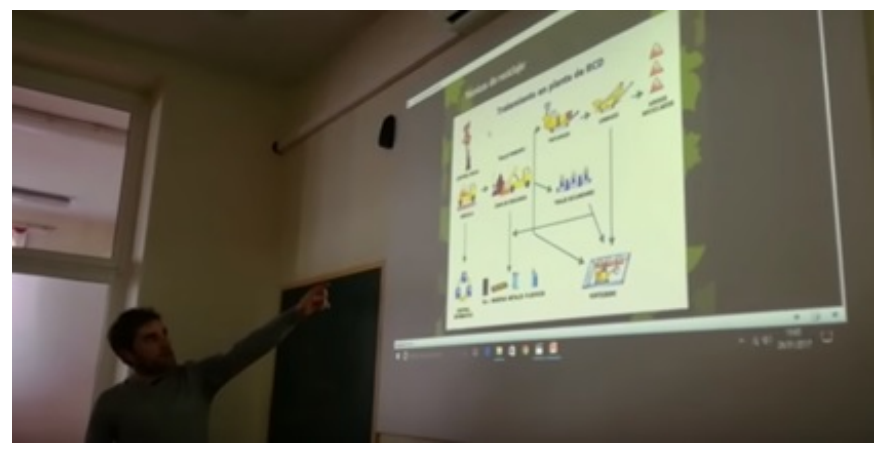

Fig. 2 Estudiante del Grado en Finanzas y Contabilidad concienciando medioambientalmente 
Los alumnos son los que seleccionan la ApS a realizar de acuerdo a sus intereses. Las ApS a su vez se seleccionan teniendo en cuenta la relacion existente entre los aprendizajes que se desean conseguir para los estudiantes con la realización del servicio y la motivación de mejorar algún aspecto concreto por parte de la entidad receptora solicitante del servicio. Esto hace que no todos los estudiantes desarrollen con la misma intensidad cada una de las competencias. Para trasladar lo aprendido al resto de compañeros, dado que las distintas ApS presentan situaciones no homogéneas, es por lo que los estudiantes exponen al resto de sus compañeros en clase lo que han hecho durante su ApS.

\section{Resultados}

Para cuantificar el impacto del aprendizaje alcanzado a través de la ApS en los estudiantes se ha elaborado un cuestionario para detectar la mejora percibida en la adquisición de competencias transversales, así como su grado de satisfacción con la actividad, en línea con estudios precedentes como el de Folgueiras et al. (2013), Ayuste et al. (2017).

Se han incorporado al mismo las siguientes cuestiones que se busca evaluar:

- Identificar el proyecto en el que ha participado el alumno.

- Tiempo total de dedicación, evaluado en horas y con carácter individual.

- Herramientas utilizadas por el grupo para la coordinación de la ApS.

- La significatividad del servicio para el estudiante.

- Grado de conexión de la ApS con el curriculum del estudiante.

- Reflexión del estudiante sobre lo alcanzado a través de la actividad, a través de distintas perspectivas.

- Grado de protagonismo del estudiante en su propia formación, en competencias, generales y específicas, y en valores.

- Adecuación del tiempo dedicado a la actividad para los objetivos alcanzados.

- Consecución de habilidades personales e interpersonales.

- Posibles áreas de mejora en la actividad.

Mediante estos ítems se pretende conocer la utilidad de la actividad realizada por el estudiante.

Las distintas habilidades profesionales, comunicativas y personales que el proyecto se plantea mejorar se trabajan integradas en la propia ApS, tal y como recoge en la Tabla 2. 
Tabla 2. Habilidades a trabajar en las ApS y forma en la que se trabajan

\begin{tabular}{ll}
\hline \multicolumn{1}{c}{ Habilidades } & \multicolumn{1}{c}{ Forma en la que se trabaja } \\
\hline $\begin{array}{l}\text { Habilidades profesionales (resolución de } \\
\text { problemas, organización, análisis y } \\
\text { procesamiento de información, } \\
\text { tecnológicas...). }\end{array}$ & $\begin{array}{l}\text { Planificando y desarrollando la propia actividad de } \\
\text { ApS }\end{array}$ \\
$\begin{array}{l}\text { Habilidades comunicativas (escucha activa, } \\
\text { asertividad, comunicación verbal, } \\
\text { comunicación no verbal, ...). }\end{array}$ & $\begin{array}{l}\text { Comunicándose con las entidades, con las personas } \\
\text { que reciben el servicio y en sus exposiciones }\end{array}$ \\
$\begin{array}{ll}\text { Habilidades personales (autoestima, seguridad, } \\
\text { inteligencia emocional, proactividad, ....). }\end{array}$ & $\begin{array}{l}\text { En si mismo se trabajan. El alumno se centra en } \\
\text { realizar la actividad, pero conforme consigue los } \\
\text { objetivos va adquiriendo seguridad en si mismo y } \\
\text { habilidades personales (disciplina, conciencia de sus } \\
\text { capacidades,...). }\end{array}$ \\
\hline
\end{tabular}

\section{Fuente: Elaboración propia}

En cuanto a la evaluación de dichas habilidades (en una escala de 0 a 7 , donde cero es totalmente en desacuerdo y 7 totalmente de acuerdo), cuya mejora se produce en un periodo corto de tiempo, concretamente en un semestre, el estudiante deberá ser el que realice su autoevaluacion para poder conocer el impacto de la ApS de cada uno de los grupos de habilidades.

Tabla 3. Valoracion de los alumnos de las ApS

\begin{tabular}{|c|c|c|c|c|}
\hline & $\begin{array}{c}\text { Oficina Verde } \\
\text { Unizar }\end{array}$ & $\begin{array}{c}\text { Fundación } \\
\text { Federico } \\
\text { Ozanam }\end{array}$ & $\begin{array}{l}\text { IES Francisco } \\
\text { Grande Covián }\end{array}$ & $\begin{array}{l}\text { YouTube } \\
\text { UNIZApS }\end{array}$ \\
\hline $\begin{array}{l}\text { Mejorar en mis habilidades } \\
\text { profesionales (resolución de } \\
\text { problemas, organización, análisis y } \\
\text { procesamiento de información, ...) }\end{array}$ & 4,14 & 4,67 & 4,25 & 4,00 \\
\hline $\begin{array}{l}\text { Mejorar en mis habilidades } \\
\text { comunicativas (escucha activa, } \\
\text { asertividad, comunicación verbal, } \\
\text { comunicación no verbal, ....) }\end{array}$ & 3,57 & 6,33 & 5,00 & 2,33 \\
\hline $\begin{array}{l}\text { Mejorar en mis habilidades } \\
\text { personales (autoestima, seguridad, } \\
\text { inteligencia emocional, proactividad, } \\
\text {...) }\end{array}$ & 2,86 & 5,50 & 4,25 & 3,00 \\
\hline
\end{tabular}

Fuente: Elaboración propia 
Aquellas ApS cuya prestación del servicio incluía una exposición pública del mismo, resaltaron que la habilidad que más habian mejorado era la comunicativa. Sin embargo, el resto de grupos habían mejorado sus habilidades profesionales (resolucion de problemas, organizacion, analisis y procesamimento de informacion, ...).

Para medir el impacto en las entidades receptoras, del mismo modo, al finalizar la actividad se realiza un cuestionario con el fin de conocer:

- $\quad$ Nombre de la organización, entidad o empresa.

- Persona que responde al cuestionario.

- Cargo.

- Servicio que ha recibido.

- Grado de satisfacción global con el servicio recibido.

- Implicación de los alumnos.

- Impacto que ha tenido el servicio en la organización.

- Adecuación de tiempo destinado a esta actividad y logro de los objetivos pretendidos.

- Habilidades personales, comunicativas, profesionales y valores que le permite al alumno alcanzar la actividad.

- Satisfacción con la organización de la actividad.

- Problemas detectados.

- Interés por volver a colaborar en un futuro

- Posibles temas para futuras colaboraciones

La opinión de la entidad receptora es valiosa no solo por la información que proporciona para la mejora de la actividad y para el engarce de la Universidad con el ámbito de la economía social, sino también porque una parte de la evaluación de la actividad es fruto del resultado de la valoración que del estudiante hace la entidad receptora de la ApS.

El impacto del proyecto ha sido positivo tanto en los estudiantes como para las entidades receptoras. En los alumnos se espera un incremento en la motivación de cara al estudio de la asignatura y a su vinculación con la sociedad que les rodea; y para las entidades receptoras ayudarles a poner en marcha una parte de sus proyectos, como en este caso será la concienciación medioambiental, formación financiera básica, formación integral, etc. 
Tabla 4. Valoracion de las entidades receptoras de las Aps

\begin{tabular}{lcccc}
\hline & $\begin{array}{c}\text { Oficina Verde } \\
\text { Unizar }\end{array}$ & $\begin{array}{c}\text { Fundación } \\
\text { Federico } \\
\text { Ozanam }\end{array}$ & $\begin{array}{c}\text { IES Francisco } \\
\text { Grande } \\
\text { Covián }\end{array}$ & $\begin{array}{c}\text { YouTube } \\
\text { UNIZApS }\end{array}$ \\
\hline $\begin{array}{l}\text { Grado de satisfaccion global } \\
\text { con el servicio recibido }\end{array}$ & 7,00 & 6,00 & 5,75 & 5,00 \\
$\begin{array}{l}\text { Implicacion de los alumnos } \\
\begin{array}{l}\text { Impacto que ha tenido el } \\
\text { servicio en la organización }\end{array}\end{array}$ & 7,50 & 6,00 & 6,00 & 5,75 \\
\hline
\end{tabular}

Fuente: Elaboración propia

La calificacion que los estudiantes obtienen en la ApS viene condicionada por varios aspectos: su exposicion en clase, si se han cumplido los objetivos marcados para la actividad y la opinion de la entidad receptora. Entendemos que si la entidad receptora no esta satisfecha con el servicio prestado la nota se ve condicionada por esta circunstancia. Esta actividad supone un $15 \%$ de la nota global de la asignatura.

Según se muestra en la Tabla 4, fue la Oficina Verde la que mejor ha valorado, en media, el servicio recibido, mientras que la peor ha valorado la actuación de los estudiantes fueron los videos de Youtube, principalmente por la baja implicación de los alumnos y porque el producto final obtenido era claramente mejorable. En una situación intermedia se encuentran el IES Grande Covián y la Fundación Federico Ozanam, en los que los valores más bajos otorgados se presentan en el impacto que ha tenido el sercicio en la propia organización.

\section{Conclusiones}

La metodología ApS promueve el aprendizaje a través de la práctica, trabajo colaborativo, grupos de discusión, etc.

En este proyecto los profesores son los que han diseñado la práctica de ApS, pero son los alumnos son los que definen y planifican su realización. Por ello, el resultado, en buena medida, depende de la motivación de los alumnos y del grado de implicación de las organizaciones participantes.

Por ello, trabajar con esta metodología supone situar al alumno en el centro, puesto que es quien elige en qué actividad participar, y los profesores pasan a ser meros facilitadores de contextos y materiales para que realmente se produzca el aprendizaje (Francisco y Moliner, 2010), y el eje en el que se sustenta la planificación de las ApS es el trabajo autónomo (Martínez-Fernandez y Rabanaque, 2008; Romero y Pérez, 2009).

La realización de este trabajo permite conocer: 1) el grado de satisfacción tanto del estudiante como de la entidad receptora de la ApS; 2) las habilidades, competencias 
Aplicación de la metodología ApS en estudiantes de Finanzas. Valoración desde la perspectiva del estudiante y de la entidad receptora de la ApS

genéricas y específicas alcanzadas por el alumno desde las dos ópticas, su propia percepción así como desde la perspectiva de la entidad receptora.

Este tipo de metodologías innovadoras no están circunscritas a unas pocas asignaturas o a un área de conocimiento determinado, sino que puede hacerse extensiva a todas las titulaciones. De este modo, se consigue que las entidades receptoras conozcan lo que los alumnos formados en la Universidad de Zaragoza les pueden aportar. Simplemente hay que encontrar la experiencia adecuada para que se desarrollen las competencias transversales y los resultados de aprendizaje vinculados a dicha asignatura. Esta sería una nueva modalidad de prácticas que se une a las tradicionales prácticas en empresas privadas, y que responden a la exigencia y demanda de un mayor compromiso social y ético.

\section{Referencias}

AYUSTE GONZALEZ, A., GROS SALVAT, B., PAYÁ SANCHEZ, M., y RUBIO SERRANO, L. (2017) "Valoración de proyectos ApS desde sus protagonistas: Un medio para la formación inicial de maestros/as" en Carrillo-Rosúa, F.J. et al. Researching The University Teaching Through ServiceLearning. Granada: Editorial Universidad de Granada, p. 35-45

CHIVA-BARTOLL, Ó., PALLARÉS-PIQUER, M., y GIL-GÓMEZ, J. (2018). “Aprendizaje-servicio y mejora de la Personalidad Eficaz en futuros docentes de Educación Física” en Revista Complutense de Educación, vol. 29 (1), p. 181-197

DANIELS, H. y BIZAR, M. (2005). Teaching the best practice way: Methods that matter. Portland, ME: Stenhouse Publishers

DELORS, J, et al. (1996). Informe a la Unesco de la comisión internacional sobre la educación para el siglo XXI: La educación encierra un tesoro. Madrid: Santillana, Ediciones UNESCO

FOLGUEIRAS BERTOMEU, P., LUNA GONZALEZ, E., y PUIG, G.(2013). “Aprendizaje y servicio: estudio del grado de satisfacción de estudiantes universitarios” en Revista de Educación, vol. 362, p. 159-185

FRANCISCO AMAT, A., y MOLINER MIRAVET, L. (2010). "El Aprendizaje Servicio en la Universidad: una estrategia en la formación de ciudadanía crítica” en Revista electrónica interuniversitaria de formación del profesorado, vol. 13 (4), p. 69-77

GIL-GÓMEZ, J., MOLINER-GARCÍA, O., CHIVA-BARTOLL, O. y GARCÍA LÓPEZ, R. (2016). "Una experiencia de aprendizaje-servicio en futuros docentes: desarrollo de la competencia social y ciudadana” en Revista Complutense de Educación, vol. 27 (1), p. 53-73

LAVE, J. y WENGER, E. (1991). Situated Learning: Legitimate Peripheral Participation. Cambridge, UK: Cambridge University Press.

MARTÍNEZ-FERNÁNDEZ, J. R. y RABANAQUE, S. (2008). “Autorregulación y trabajo autónomo del estudiante en una actividad de aprendizaje basada en las TIC” en Anuario de Psicología, vol. 39 (3), p. 311-331.

MAYOR PAREDES, D. M. (2012). “Aprender realizando una actividad social. Guía básica para el diseño de proyectos de Aprendizaje-Servicio" en RES: Revista de Educación Social, vol. 16, p. 1-14

PÉREZ GÓMEZ, A.I. (2010). "La naturaleza del conocimiento práctico y sus implicaciones en la formación de docentes” en Infancia y aprendizaje, vol. 33(2), p. 171-177

(cc) EY-NC-ND 2018, UniversitatPolitècnica de València

Congreso IN-RED (2018) 
PÉREZ GÓMEZ, A.I. y SOTO GÓMEZ, E. (2009). “Competencias y contextos escolares. Implicaciones mutuas” en Organización y Gestión Educativa: Revista del Fórum Europeo de Administradores de la Educación, vol. 17 (2), p. 17-22

RODRÍGUEZ GALLEGO, M. R. (2014). “El Aprendizaje-Servicio como estrategia metodológica en la universidad” en Revista Complutense de Educación, vol. 25 (1), p. 95-113

ROMERO ARIZA, M. y PÉREZ FERRA, M. (2009). “Cómo motivar a aprender en la universidad: una estrategia fundamental contra el fracaso académico en los nuevos modelos educativos” en Revista Iberoamericana de Educación, vol. 51, p. 87-105

SAZ GIL, I.; RAMO GARZARÁN, R.M. (2015) “Aproximación a los impactos y beneficios del aprendizaje servicio en la Universidad de Zaragoza” en. RIDAS, Revista Iberoamericana de Aprendizaje y Servicio, vol. 1, p. 9-27

WONG, D. (2007). "Beyond control and rationality: Dewey, aesthetics, motivation, and educative experiences” en Teachers College Record, vol. 109 (1), p. 192-220 